\title{
Three-dimensional Finite Element Modelling of the Cylindrical Specimen Upsetting
}

\author{
Mikhail V. Murashov ${ }^{1}$, Andrey V. Vlasov ${ }^{1}$ \\ ${ }^{1}$ Bauman Moscow State Technical University, 105005 Moscow, Russia
}

\begin{abstract}
Friction has a substantial influence on the metal forming at upsetting of cylindrical aluminum specimens. The finite element method is often used to investigate this problem. This paper aims to reveal possible numerical errors and obstacles related to the 3D finite element solution of the problem. The calculation results for the proposed numerical 3D-model are compared with the experimental data. The influence of friction is demonstrated and a good agreement on the tool displacement is obtained. The features of the numerical solution of the problem in the ANSYS finite element software are shown.
\end{abstract}

\section{Introduction}

The friction coefficient has a significant effect on the metal forming of parts, in which the simplest problem is the upsetting of a cylindrical specimen. On this problem, the friction processes in metal forming are usually studied [1]. There are a number of works on semi-empirical methods of shape characteristics calculation for the upsetting process [2-4]. However, numerical methods are more versatile and consequently accurate. The finite element method is widely used for the calculation of elastoplastic deformation of parts, including for the upsetting problem. For example, the problem of upsetting of a cylindrical sample has been solved in a twodimensional formulation for nearly half a century [5-8]. Finite element based work [9] is devoted to the determination of the hardness distribution of a sample after deformation using optimization software. A finiteelement 3D-model was used to solve the inverse problem of obtaining a barrel-independent stress-strain curve from experimental data in [10]. At the same time, the possible significant influence of errors in numerical simulation is still in question.

Friction plays an important role in shaping when upsetting of cylindrical sample, namely, it results in the formation of a barrel. Not knowing the friction coefficient, you cannot predict the upsetting results. There have been many attempts to relate the curvature of the barrel to friction coefficient [11]. It should not be forgotten that in reality the friction coefficient is not constant in the upsetting process and is different at different points of contact. Numerical simulation of deformation, performed after finding friction coefficient and stress-strain curve from such an experiment, can cause specific difficulties. In this paper it is shown that in a finite-element 3D solution for a certain values of the friction coefficient the calculation takes long time to converge or may fail at all.

The purpose of this paper is to investigate the errors in the numerical 3D modeling of upsetting of an aluminum cylindrical specimen according to the stressstrain curve determined from the experiment. The problem was solved by the finite element software ANSYS. The comparison of the upsetting experiment data with the data of numerical modeling confirms also a possibility of ANSYS to calculate the elastic-plastic deformation at large strains. In order to exclude the mutual influence of different factors, the very technique of the upsetting experiment for obtaining a stress-strain curve is modeled. The strains distribution in the section of the deformed sample was also obtained.

\section{The model description}

The finite element software ANSYS has been previously tested by the manufacturer on known analytical solutions for a range of elastic contact problems. The following test examples are listed in the documentation to the program: - the test example VM63 shows that the error in solution of Hertz problem of elastic contact of the sphere with the rigid plane [12] for contact spot radius is $0.1 \%$;

- the test example VM191 shows that the errors in solution of Hertz problem of elastic contact of two cylinders [13] for the approach distance does not exceed $0.2 \%$ and for the semi-contact length $-3.3 \%$;

- the test example VM272 shows the error in solution of problem of elastic contact with friction of two half cylinders [14] for contact pressure is less than $3 \%$ and for friction contact stress does not exceed 7\%;

- the test example VM292 shows the error in determination of contact pressure at elastic connection of 
two hollow cylinders with an interference fit between them to be $1 \%$ [15].

The test examples assume only elastic behavior of material. We note that an experiment on the indentation of a rigid ball into a deformable plane surface was considered by Shankar and Mayuram [16]. That work shows that elastic-plastic calculation with multilinear hardening in ANSYS gave results in a high degree of accuracy coinciding with the experiment. On the contrary, this paper shows the solution of the elastic-plastic problem with hardening of upsetting of deformable body by a rigid plane.

\subsection{Physical experiment}

To estimate the accuracy of the finite element solution there was performed the comparison of results of a physical upsetting experiment with the results of corresponding numerical modeling. From the upsetting experiment of the cylindrical specimen made of aluminum 1050 UNS A91050 the stress-strain curve was acquired [17]. The experiment was carried out according to ASTM E9 standard. The following average values were received from testing of five specimens $23.6 \mathrm{~mm}$ high and $15.1 \mathrm{~mm}$ in diameter: the displacement of the tool is $14.787 \mathrm{~mm}$ at the maximum pressure of $80002 \mathrm{~N}$, and $14.296 \mathrm{~mm}$ after unloading. The friction coefficient was not defined in this experiment.

\subsection{D finite element model}

In numerical model it is impossible to apply pressure to the upper surface of the cylinder as at compression the cylinder widens, the area of the upper surface increases, the force grows respectively thus resulting in sharp, physically improbable growth of displacement with distortion of finite elements. So pressure is applied to a non-deformable plate of constant area which in turn transfers pressure to the cylinder. The bottom of the cylinder is installed on a fixed non-deformable plate.

Let us consider a 3D quasi-static mathematical model of the contact of two plates and the cylinder with volumes $V_{1}, V_{2}, V_{3}$, limited by surfaces $S_{1}, S_{2}, S_{3}$, respectively (see Figure 1(a)). We pass to the designation of the Cartesian coordinate system axes with the use of subscripts $x_{1}, x_{2}$, $x_{3}$. For the first body we designate the upper flat surface $S_{1}^{1}$ and the rest of the surface $S_{1}^{2}$, so that $S_{1}=S_{1}^{1} \cup S_{1}^{2}$. For the second body we designate the contacting part of the surface $S_{2}^{1}$ and the rest of the surface $S_{2}^{2}$, so that $S_{2}=S_{2}^{1} \cup S_{2}^{2}$.

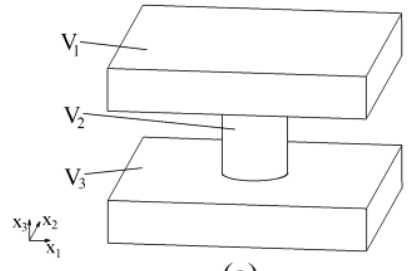

(a)

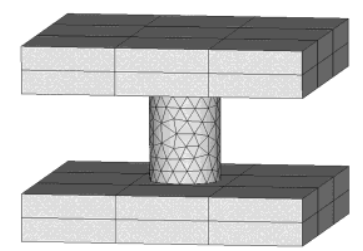

(b)
Figure 1. The models for compression test: (a) geometrical; (b) finite element.

The surface $S_{1}^{1}$ is affected by the external pressure $P$. The mathematical model comprises equilibrium equations
(1), the generalized Hooke's law (2), the flow rule (3), strain-displacement relations (4), von Mises yield condition (5), ratio for calculation of contact pressure of augmented Lagrangian method (6), Amontons-Coulomb friction law (7) on the contact surface, and boundary conditions (8)-(10)

$$
\begin{gathered}
\sigma_{j i, j}=0 ; \\
\varepsilon_{i j}=\frac{1+v}{E} \sigma_{i j}-\frac{v}{E} \delta_{i j} \sigma_{k k} ; \\
d \varepsilon_{i j}^{P}=s_{i j} d \lambda ; \\
\varepsilon_{i j}=\frac{1}{2}\left(u_{i, j}+u_{j, i}\right) \\
\left(\sigma_{1}-\sigma_{2}\right)^{2}+\left(\sigma_{2}-\sigma_{3}\right)^{2}+\left(\sigma_{3}-\sigma_{1}\right)^{2}=2 \Phi\left(\varepsilon^{P}\right)^{2} ; \\
p\left(x_{i}\right)=K \delta+\lambda_{c}, x_{i} \in S_{2}^{1} ; \\
\tau_{c} \leq \mu p\left(x_{i}\right), x_{i} \in S_{2}^{1}, \\
\sigma_{33}=P, x_{3} \in S_{1}^{1}, \\
u_{1}\left(x_{2}\right)=0, u_{2}\left(x_{1}\right)=0, x_{1}, x_{2} \in S_{1}^{2}, \\
u_{i}\left(x_{i}\right)=0, x_{i} \in V_{3},
\end{gathered}
$$

where $\sigma_{i j}$ and $\varepsilon_{i j}$ are the Cartesian components of tensors of stress and strain, $u_{i}$ - components of displacements vector, $E-$ the modulus of elasticity, $v$ - Poisson's ratio, $\delta_{i j}$ - Kronecker delta, $s_{i j}$ - components of stress deviator tensor, $\sigma_{1}, \sigma_{2}, \sigma_{3}-$ the principal stresses, $\lambda, \lambda_{c}$ - Lagrange multipliers, $\Phi\left(\varepsilon^{P}\right)$ - function of the material's stressstrain curve, $p$ - the contact pressure, $P$ - external pressure applied to the punch, $K$ - contact stiffness, $\delta$ contact gap size, $\tau_{c}-$ tangential stress on the contact surface, $\mu$ - friction coefficient.

The contact finite elements CONTA174 cover the whole surface of the cylinder, and finite elements TARGE170 cover the contacting surfaces of plates. The finite element model is shown in Figure 1(b).

Materials of bodies are assumed homogeneous and isotropic. In this paper the plates are considered made of a hypothetical material with the modulus of elasticity $10^{18}$ $\mathrm{GPa}$ and Poisson's ratio of 0.3 . Material of the cylinder is elastic-plastic with isotropic hardening based on multilinear approximation of the stress-strain curve received in physical experiment (see Figure 2).

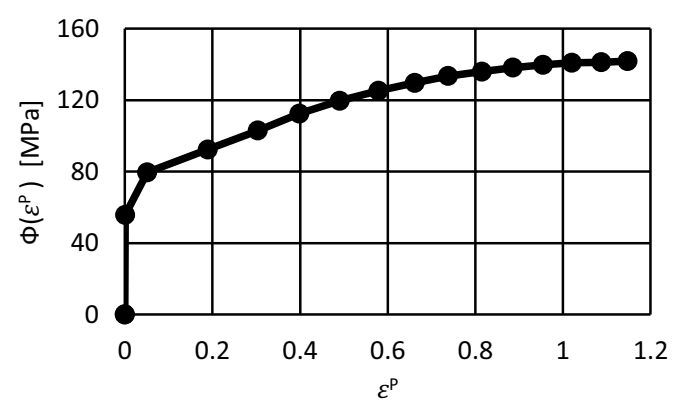

Figure 2. The adopted in this work multilinear approximation of stress-strain curve for aluminum 1050 UNS A91050 at compression.

Assumptions of the numerical model: 
- the model has no grooves at tops of the cylinder and their influence is neglected;

- the friction coefficient is accepted identical to all contacting surfaces and does not change in the course of deformation;

- compliance of the press is neglected;

- the isotropic friction between the contacting surfaces obeys Amontons-Coulomb friction law;

- influence of the surfaces roughness, lubrication and temperature is neglected.

\section{Discussing the results}

Solution results for the friction coefficient of 0.01 and 0.6 are shown in Figure 3,4. Results show an adequate picture of increase in irregularity of strains with increase in friction coefficient.
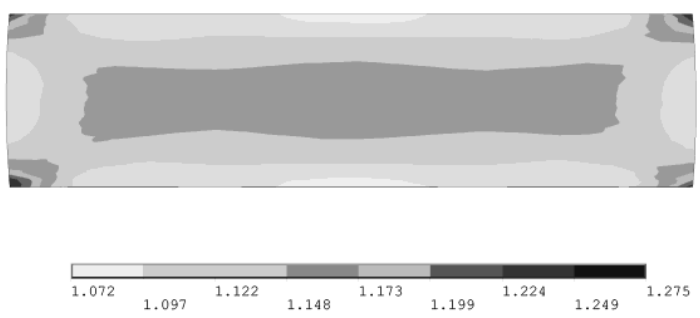

Figure 3. Von Mises or equivalent strains in the axial section of the cylinder at the friction coefficient of 0.01 (48866 nodes mesh).
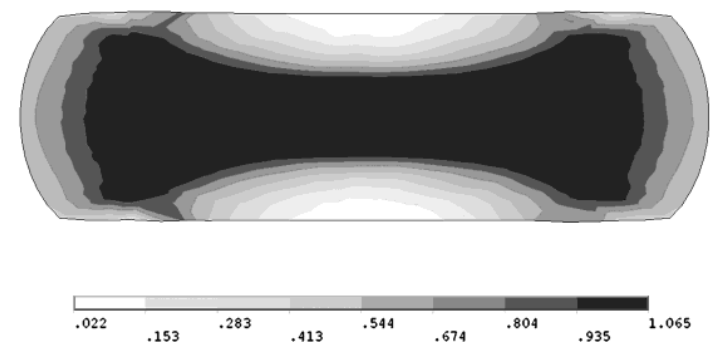

Figure 4. Von Mises or equivalent strains in the axial section of the cylinder at the friction coefficient of 0.6 (48866 nodes mesh).

According to ASTM E9 the efforts are made to decrease friction in the experiment and it is possible to assume friction coefficient to be close to 0 . However, to check the influence of friction on the result, the calculation was carried out for a number of the friction coefficient values in the range of $0 \ldots 0.5$. Pressure in the contact area reached $1 \mathrm{GPa}$ and more at $\mu=0.5$, because of which the friction stress significantly exceeded the actual yield stress of the material. Therefore, in addition to Coulomb friction model there is imposed a restriction that the friction stress cannot exceed the yield stress of the material. As ANSYS has no standard function of binding the friction stress limitation to the current value of yield stress, so, as a first approximation, the friction stress is limited to a constant value equal to the maximum tangential yield stress taking place in the course of deformation. According to the stress-strain curve (Figure 2) the maximum achievable yield stress makes $141 \mathrm{MPa}$ therefore the friction value of $141 / \sqrt{3}=82 \mathrm{MPa}$ is accepted as the maximum tangential friction stress.
The value of the upper plate (which is model of tool) displacement at application of external pressure equivalent to force of $80002 \mathrm{~N}$ depending on the assigned friction coefficient is shown in Figure 5. Small deviations of the graphs from each other are due to the numerical features of the operation of contact algorithms and contact detection methods. The results are obtained by using the pure Lagrange method as a contact algorithm with "nodes to target" contact detection method. Another strategy is the use of a combination of augmented Lagrange method as a contact algorithm with Gauss point contact detection method. In the latter case the difference in the results is within $1 \%$.

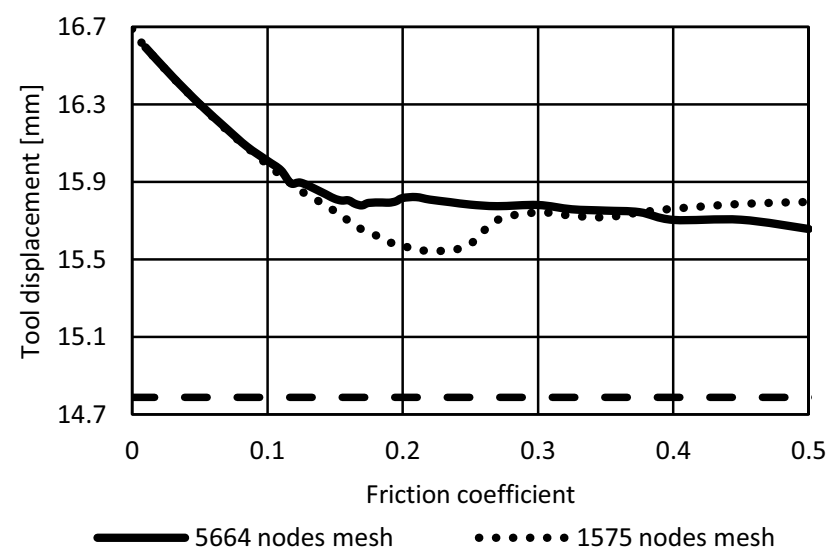

Figure 5. Displacement on $x_{3}$-axis of the upper plate depending on the friction coefficient.

To reveal the influence of density of the finite element mesh the calculations in the ANSYS software were carried out for two cases of uniform mesh. The finite element models are comprised of 1575 and 5664 nodes. It can be seen that the fluctuations of the graphs in a range of friction coefficients $0.1-0.3$ become smaller with the mesh refinement. In general, these fluctuations relate to domain discretization and do not exceed $2 \%$ of tool displacement that can be deemed not considerable, and do not change qualitatively the physically reasonable tendency to decrease in tool displacement with increase in friction coefficient. Nevertheless, after mesh refinement to 48866 nodes, the calculation in the range of friction coefficients of 0.1-0.22 failed due to the lack of convergence.

The provided graph is somewhat artificial as in reality the friction coefficient changes in the process of compression and differs along the lateral direction of contact area $[8,18]$. Nevertheless, the obtained results (see Figure 5) allow supposing weak influence of the friction coefficient on the value of the tool displacement (less than $8 \%$ ). The maximum displacement of the tool for the considered range of friction coefficients from 0 to 0.5 deviates from the experimental value by no more than $13 \%$. Taking into account a number of accepted assumptions considerably idealizing the problem, it is possible to view the obtained results as an additional confirmation of the possibility to carry out modeling of elastic-plastic deformation with the use of finite element software ANSYS. 


\section{Conclusion}

Numerical investigation of the spatial problem of the upsetting of a cylindrical sample is carried out. As expected, the shape of the model after deformation (barrel shape) essentially depends on the friction coefficient. The strain distribution in the section of the deformed model is determined. The calculated value of the tool displacement was predicted with an appropriate accuracy. Thus, on the one hand, elastoplastic calculations in ANSYS are reliably performed when it is necessary to determine the tool displacement. On the other hand, to calculate accurately the final shape of a part, it is necessary to know the coefficient of friction, some values of which can lead to erroneous solution fluctuations or even the solution failure.

\section{References}

1. T. Yoneyama, K. Miyoshi, T. Tate, J. Manuf. Process. 32, 791-801 (2018)

2. J.A. Schey, T.R. Venner, S.L. Takomana, J. Eng. Ind. 104(1), 79-83 (1982)

3. S. Malayappan, G. Esakkimuthu, Int. J. Adv. Manuf. Technol. 29, 41-48 (2006)

4. C.S. Çetinarslan, Mater. Des. 28(6), 1907-1913 (2007)

5. A. Nagamatsu, T. Murota, T. Jimma, Bull. JSME 14, 339-347 (1971)
6. K.D. Vertin, S.A. Majlessi, J. Eng. Ind. 115(4), 450458 (1993)

7. X. Tan, Proc. Instn. Mech. Engrs. Part J 215, 189200 (2001)

8. A. Azushimaa, Sh. Yoneyamaa, H. Utsunomiya, Wear 286-287, 3-7 (2012)

9. Ch. HariKrishna, M.J. Davidson, Ch. Nagaraju, Trans. Indian Inst. Met. 68(2), 219-228 (2015)

10. X. Wang, H. Li, K. Chandrashekhara, S.A. Rummel, S. Lekakh, D.C. Van Aken, R.J. O’Malley, J. Mater. Process. Technol. 243, 465-473 (2017)

11. X.G. Fan, Y.D. Dong, H. Yang, P.F. Gao, M. Zhan, J. Mater. Process. Technol. 243, 282-290 (2017)

12. S. Timoshenko, J.N. Goodier, Theory of Elasticity (McGraw-Hill Book Co. Inc., New York, 1970)

13. N. Chandrasekaran, W.E. Haisler, R. E. Goforth RE, Finite Elem. Anal. Des. 3(1), 39-56 (1987)

14. B. Yang, T.A. Laursen, X. Meng, Int. J. Numer. Methods Eng. 62(9), 1183-1225 (2005)

15. R.G. Budynas, J.K. Nisbett, Shigley's Mechanical Engineering Design (McGraw-Hill Education, New York, 2015)

16. S. Shankar, M.M. Mayuram, Int. J. Solids Struct. 45(10), 3009-3020 (2008)

17. M.V. Murashov, Y.V. Kornev, Tech. Phys. 84(3), 75-81 (2014)

18. X. Tan, Prod. Eng. Res. Devel. 5 141-149 (2011) 